\title{
Overview of the relationship between pain and obesity: What do we know? Where do we go next?
}

\author{
E. Amy Janke, PhD; ${ }^{1^{*}}$ Allison Collins, PhD; ${ }^{2}$ Andrea T. Kozak, $\mathbf{P h D}^{3-4}$ \\ ${ }^{1}$ Edward Hines Jr Department of Veterans Affairs (VA) Hospital, Center for Management of Complex Chronic Care, Hines, \\ $I L ;{ }^{2}$ VA Boston Healthcare System, Psychology Service, Boston, MA; ${ }^{3}$ Department of Preventive Medicine, Feinberg \\ School of Medicine, Northwestern University, Chicago, IL; ${ }^{4}$ Edward Hines Jr VA Hospital, Research Service, Hines, IL
}

\begin{abstract}
Overweight/obesity and pain are significant problems of increasing prevalence; understanding the relationship between the two is the focus of a growing body of research. Findings from this review support a likely relationship between increasing weight and pain conditions such as osteoarthritis and low back pain. Longitudinal studies suggest overweight/obesity early in life is a risk factor for pain, and the co-occurrence of pain and overweight/obesity negatively affects quality of life. The mechanism of relationship is unknown but is hypothesized to include mechanical and metabolic abnormalities, possibly secondary to lifestyle choices. Observations from a few studies demonstrate that treatments for obesity reduce pain secondary to weight loss. Few studies examine both pain and weight as primary outcomes, and variability in measurement makes comparisons and conclusions difficult. Research should focus on expanding knowledge about mechanisms of the relationship between pain and obesity, testing explanatory models addressing their co-occurrence, and developing treatments that most effectively target this comorbidity.
\end{abstract}

Key words: back pain, body mass index, body weight, literature review, obesity, osteoarthritis, pain, quality of life, rehabilitation, risk factors, weight loss.

\section{INTRODUCTION}

Overweight/obesity is a growing epidemic within the United States and, in particular, a substantial burden among veterans who use Department of Veterans Affairs (VA) medical facilities. An expensive condition that is associated with significant disability [1], overweight/obesity is the leading cause of preventable deaths and chronic disease [2]. Comorbidities frequently associated with overweight/ obesity include diabetes, hypertension, and coronary heart disease, and a growing body of literature describes the association between overweight/obesity and pain. Given the importance of understanding the relationship between weight and pain from both a public health perspective and specifically in terms of health outcomes for the Veterans Health Administration (VHA), we conducted a literature review to appraise the current state of the science and provide recommendations for future research.

Thus, our goal was twofold. First, we aimed to review available literature that examined the relationship between weight and pain. This review focused on (1) the pain

\footnotetext{
Abbreviations: ADAPT $=$ Arthritis, Diet, and Activity Promotion Trial, BMI = body mass index, FMS = fibromyalgia syndrome, HRQoL = health-related quality of life, LBP = low back pain, NHANES = National Health and Nutrition Examination Survey, OA = osteoarthritis, RCT = randomized clinical trial, SF-36 = 36-item Short Form (Health Survey), VA = Department of Veterans Affairs, VHA = Veterans Health Administration, VLED = very-low-energy diet.

* Address all correspondence to E. Amy Janke, PhD; Center for Management of Complex Chronic Care, 151H, Edward Hines Jr VA Hospital, PO Box 5000, Hines, IL 60141; 708202-2838; fax: 708-202-2316. Email: elizabeth.janke@va.gov DOI: 10.1682/JRRD.2006.06.0060
} 
conditions, such as osteoarthritis (OA) and low back pain (LBP), most frequently researched in co-occurrence with overweight/obesity; (2) the relationship between healthrelated quality of life (HRQoL), pain, and obesity; (3) the effects of weight reduction on pain outcomes and of pain treatments on weight loss; and (4) possible mechanisms to explain the co-occurrence of pain and obesity. Second, we hoped this review would pinpoint areas where further research is needed to better understand and develop effective treatments that target the co-occurrence of these two disorders.

\section{METHODS}

We conducted literature searches using MEDLINE/ PubMed to obtain relevant studies. We did not limit the search parameters to specific publication years; however, we preferred to include and review articles published in the year 2000 or later (until March 2006). The initial search included broad index terms: obesity, body mass index, $B M I$, weight, and pain. To further narrow the scope of this review, we performed subsequent specialized searches by combining the original search terms with additional keywords, including arthritis, osteoarthritis, fibromyalgia, headache, neuropathy, quality of life, back pain, weight loss, and treatment. Articles were also obtained from reference lists of research reports and reviews. Articles most relevant to our objectives were included in this review. This review provides a breadth of discussion regarding the most substantially researched areas of pain and obesity and not necessarily a detailed systematic review of the literature within this area. When the breadth of literature available in a topic area exceeded the limitations of this review, articles that were the most representative of work conducted within that area with the highest methodological quality were chosen for inclusion (e.g., criteria that would lead to exclusion might include small sample size, nonrepresentative samples, and assessment of weight alone without providing data on weight relative to height).

\section{Prevalence of Pain and Overweight/Obesity Among Veterans}

Pain is one of the most common complaints reported to healthcare providers. Findings from a recent examination among veterans who received primary care services at one VA facility suggest that the prevalence of pain and its associated negative outcomes is high. In a sample of 685 veterans who were seeking primary care services,
Kerns and colleagues found that nearly 50 percent regularly reported experiencing pain and associated concerns [3]. In comparison with veterans who did not report pain, those who experienced regular pain had greater emotional distress, reported worsening health, were more likely to smoke, had greater diet and weight-related concerns, and used more outpatient medical services.

Overweight/obesity is a significant public health concern associated with increased morbidity and mortality $[2,4]$. The National Institutes of Health and the World Health Organization endorse the use of body mass index (BMI) (kilogram per square meter) to define obesity as BMI $\geq 30 \mathrm{~kg} / \mathrm{m}^{2}$ and overweight as BMI $\geq 25 \mathrm{~kg} / \mathrm{m}^{2}$ [2], classifications based on epidemiological evidence linking increasing weight to increasing risk of weight-related comorbidities and premature mortality [4]. Prevalence rates of overweight/obesity among adults in the United States have increased markedly during the last decade, with current rates estimated at 66.3 percent for overweight and 32.2 percent for obesity [5]. Rates of obesity within the VHA are estimated to be higher than these national figures. In a recent study that examined the prevalence of obesity in more than 1.8 million veterans who received care at 136 VA medical facilities, Das and colleagues concluded that obesity is a substantial problem within the VA for both males and females [6]. Among men, 73.0 percent were at least overweight, 32.9 percent were obese, and 3.3 percent were classified as class-III obese (i.e., BMI $\geq 40$ $\mathrm{kg} / \mathrm{m}^{2}$ ). Among female veterans, nearly 68.5 percent were at least overweight, 37.4 percent were obese, and 6.0 percent were class-III obese.

\section{Pain Conditions Associated with Overweight/Obesity}

As the epidemic of overweight/obesity in the United States and abroad gains increasing attention, investigators are beginning to examine the effects of higher BMI on a variety of conditions. In the area of pain specifically, researchers have begun to examine whether increased weight may be associated with conditions including headache [7], fibromyalgia [8], and rheumatoid arthritis [9]. Yet, perhaps in part because the problem of overweight/ obesity and its association with pain is such a new question, few studies have been published to date. Thus, given the early state of the evidence with regard to many of these pain conditions, review and summary conclusions are tenuous at best. Of the available research that examined the cooccurrence of pain and overweight/obesity, musculoskeletal pain has received the most attention. In particular, two 
conditions stand out as the most frequently examinedLBP and OA.

\section{Low Back Pain}

LBP is among the most common chronic pain conditions. Untreated, it can cause significant personal suffering as well as notable economic consequences at both the societal and individual level [10-11]. Symptoms of chronic LBP are varied - indeed, LBP is often a symptom rather than a discrete diagnosis in and of itself-and pain may be experienced in one position or many positions and may vary in intensity and interference over time [12]. LBP is typically defined as acute when lasting less than 6 weeks, subacute when lasting between 6 weeks and 3 months, and chronic when lasting more than 3 months. And, while many patients with LBP recover quickly, LBP commonly follows a recurrent course, with exacerbations occurring over time. Prevalence of LBP varies according to definitions used and populations studied. Generally, lifetime prevalence rates of LBP are estimated to be 70 to 85 percent and point prevalence rates average around 30 percent [13]. Risk factors for the presence and severity of LBP include both sociodemographic factors, such as age [14], as well as lifestyle factors, such as smoking and physical conditioning [15-16]. Additionally, a growing body of research questions whether excess body weight is a likely risk factor for LBP.

A remarkable number of studies have been published that attempt to address the nature of the relationship between LBP and overweight/obesity, and equally remarkable given these attempts is the lack of conclusive evidence elucidating the link between weight and LBP. Simply stated, while intuitively LBP and excess weight could be related, the current state of science is such that we cannot determine whether they are in fact directly related, under what circumstances they are related, how they become related, the strength of relationship (if one does in fact exist), and the impact of a change in one condition on the other. Some cross-sectional studies conducted in general populations have found no association between LBP and increasing BMI [17-18], while others have found a positive association between the two [1920]. Several studies have found LBP to be independently associated with increased BMI in large $(N>5,000)$ crosssectional samples of adults from the general population [21-22]. A large nationwide study of 15,974 patients with common spine diagnoses found that higher BMI in this population was associated with increased disability, more severe pain symptoms, and more comorbidities than in the nonobese spine patient population [23]. Combined, studies such as these that report a positive association between overweight/obesity and LBP suggest a possible doseresponse relationship between the two such that increasing prevalence of LBP occurs with increasing BMI. However, other large cross-sectional studies have found more equivocal results. Leboeuf-Yde and colleagues found a modest positive association between obesity and LBP, in particular chronic LBP, in a cross-sectional survey of 29,424 twin subjects [24]. However, their findings that the relationship between obesity and LBP was "A-shaped," such that increased obesity levels were "less dangerous," that the relationship between obesity and LBP disappeared in monozygotic twin pairs who were dissimilar in obesity status, and that the overall positive association was weak suggest no direct causal explanation exists [24].

The few longitudinal studies available to date that have examined the causal association between overweight/obesity and LBP do not clarify the matter. For example, Aro and Leino found no relationship between weight and LBP in a 10-year follow-up study of Finnish adults [25]. In Aro and Leino's study, no association was reported between baseline weight or change in weight and LBP during the 10 years of follow-up. More recently, Lake and colleagues found some evidence in a longitudinal cohort study that obesity at age 23 increased risk of LBP onset for women, but not men, 10 years later [26]. Most interestingly, Lake and colleagues found that other lifestyle factors—such as smoking — had a confounding effect on their analyses. These findings led them to suggest that the relationship between LBP and weight is not necessarily directly causal but is, at least in part, secondary to lifestyle changes associated with unhealthy behaviors and poor overall health.

Several recent reviews have attempted to address the relationship between LBP and weight. Generally, these reviews have suggested that, while a relationship between overweight/obesity and LBP may indeed exist, the current state of science lacks evidence clearly establishing a direct relationship between the two. Garzillo and Garzillo, in an early review of seven studies, found no evidence to support the recommendation of weight loss to treat LBP, though they did state that some evidence of a correlational relationship existed between LBP and BMI $>29 \mathrm{~kg} / \mathrm{m}^{2}$ [27]. More recently, Leboeuf-Yde conducted a systematic review of the epidemiological literature to establish whether weight is causally associated with LBP [28]. In 
this review of 65 studies, findings were inconsistent and the conclusion was that not enough evidence existed to determine whether a causal relationship existed between body weight and LBP. A positive association between body weight and LBP was found in only 32 percent of studies, and the association between weight and LBP, when present, was found to be weak. Finally, Mirtz and Greene conducted a review of recent evidence regarding the relationship between overweight/obesity and LBP and similarly concluded that while current data do not provide a clear answer for untangling the relationship between overweight/obesity and LBP, results suggest that individuals with $\mathrm{BMI}<30$ are at minimal risk of developing LBP, those between 30 and 40 are at moderate risk, and patients with BMI $>40$ are at high risk [29].

So, the question remains: How can we know so much about overweight/obesity and LBP and still know so little? One answer to this question is that perhaps the relationship between the two is much weaker than previously hypothesized. A direct causal relationship between weight and LBP may or may not exist; indeed Leboeuf-Yde et al.'s meta-analysis certainly suggests that the relationship, if present, is weak [24]. Another answer is that methodological issues may be obscuring what could be a weak relationship in the first place. For example, some of the difficulty may stem from the fact that LBP is often a nonspecific disease-the probability that a particular case of LBP has a specific cause that can be identified on radiographic examination is estimated at $<1$ percent [30]. Most cases of LBP have no identifiable cause and no reliable and valid classification system. This lack of diagnostic certainty is reflected in the studies that examined weight and pain but used widely varying definitions of LBP. Some studies used self-reported pain, others required clinical assessment and diagnosis, and measurements of pain frequency and chronicity varied greatly. With no standard definition available, conclusions about the relationship between LBP and overweight/obesity are challenging.

Also, many of these studies included weight as one variable among many believed to affect LBP. This is a useful approach for early exploratory work; however, few studies to date have approached the examination of weight and LBP with specific testable hypotheses. Rather, many of these studies continue to examine the relationship between weight and LBP in secondary analysis or as part of exploratory research. To move research forward in this area, researchers need prospective hypothesis-driven research that tests specific questions regarding the relationship between weight and LBP [28,31].

Finally, inconclusive findings may point to the importance of other mediating factors that affect the relationship between weight and pain. Indeed, as has been shown in some of the research to date, the relationship between overweight/obesity and LBP may not be directly causal. Some of the factors that could function as possible mediators and need further investigation include sex [26,32], age [19], height/stature [31,33], distribution of body fat and waist circumference [17,34-35], socioeconomic factors [36], and lifestyle factors (smoking [15,21,26] and physical activity [37-38]). This list is by no means exhaustive, and other factors shown to have high correlations with both overweight/obesity and LBP may also be likely candidates for examination. What is clear is that more work needs to be done to examine possible common pathways between overweight/obesity and LBP to further clarify the relationship between the two.

\section{Summary: Relationship Between Overweight/Obesity and Low Back Pain}

Many studies-both cross-sectional and longitudinal-have examined the relationship between weight and LBP, but whether, how, and why these are related is still unclear. More specific research is needed to understand this relationship.

Why don't we know more?

- LBP is often a nonspecific disease.

- Studies use different definitions of LBP.

- Relationship may not be direct, but rather mediated by other factors such as lifestyle (e.g., smoking, physical activity).

- Relationship between the two may be weaker than initially hypothesized.

\section{Osteoarthritis}

Some of the earliest research examining the relationship between weight and pain focused on OA, in particular OA of the knee and hip. OA is the most common joint disorder, affecting more than 25 million Americans [39], and the leading cause of disability among older Americans [40]. Clinically, it is characterized by joint pain, tenderness, limitation of movement, and variable degrees of local inflammation. The course of the disease varies, but is often progressive, and the condition is not reversible. OA of the knee and of the hip are among the most disabling sites and result in a loss of mobility and more than 
250,000 joint replacements each year. Approximately 10 to 13 percent of persons over age 65 are estimated to have symptomatic knee OA, though more than 30 percent are estimated to have radiographic findings of knee OA [41]. Prevalence of symptomatic hip OA is estimated at approximately 3 percent, with prevalence of radiographic hip OA averaging 5 to 10 percent [39].

High BMI has been shown to be a likely risk factor for development and progression of OA in the knee and hip, and possibly development of OA in the hand. Of these three sites, overweight/obesity is most clearly and consistently studied, linked, and confirmed with OA of the knee. The evidence is strong enough that the American College of Rheumatology OA treatment guidelines recommend weight loss for overweight persons with knee OA [42]. Obesity has been shown to be strongly associated with knee OA across race/ethnicity and sex [43-45], although some conflicting evidence about the relative strength of the association in males versus females exists [46]. Analyses conducted on the National Health and Nutrition Examination Survey (NHANES) data show that adults in the United States with a BMI $\geq 30 \mathrm{~kg} / \mathrm{m}^{2}$ have a $>4$-fold higher prevalence of radiographic knee OA than those with a BMI $<30 \mathrm{~kg} / \mathrm{m}^{2}$ [47]. Similarly, longitudinal studies have demonstrated that increased BMI in both young and middle aged adults increases risk of subsequent knee OA. In one study, the risk of developing both radiographic and symptomatic knee OA was increased in older adults who were obese an average of 37 years earlier in life [48]. In another study, greater BMI in young men aged 20 to 29 was associated with an increased risk of subsequent knee OA later in life [49]. Of note, while those in the highest BMI categories had a greater risk than those in the lowest categories, no threshold effect was found, which suggests that even modest to moderate levels of overweight-less than conventional definitions of obesity-convey increased risk. Even within the normal weight range of BMI $<25 \mathrm{~kg} / \mathrm{m}^{2}$, moderate increases in BMI have been shown to be significantly related to knee OA [46], which suggests that even modest levels of increasing weight convey an increased risk of knee OA.

While evidence supporting a link between increasing BMI and knee OA is fairly strong, in comparison, studies examining the relationship between overweight/obesity and hip OA provide somewhat conflicting evidence. For example, in a longitudinal study examining whether increased BMI in young men was a risk factor for subsequent development of OA of the knee and hip, Gelber and colleagues found that higher BMI was associated with an increased risk for knee, but not hip, OA [49]. One limitation of this and many other studies that examined the link between OA and weight was the use of selfreported diagnoses and a lack of radiographic confirmation. In the NHANES, where detailed arthritis examinations included X-rays and physical examinations, obesity was found to be significantly associated with OA of the hips in white women and nonwhite males only [43]. More recently, a large cohort study that matched BMI data from more than 1 million persons to a national arthroplasty register found a strong dose-response association between BMI and later total arthroplasty for hip OA in both males and females [50]. In particular, the study found that increased weight while young (compared with increased weight when older) had a greater affect on arthroplasty rates. However, this study did not include information on other known risk factors for total hip arthroplasty, such as physical activity, joint injury, and heredity, thus the true impact of BMI on arthroplasty rates remains unclear. Additionally, as this study was conducted within a publicly financed system, access to healthcare could also have biased the end results. Thus, while evidence to date suggests a likely link between increasing weight and hip OA, further examination is necessary to clarify what other risk factors may contribute to the relationship.

Finally, a small number of studies suggest a possible link between increasing weight and OA of the hand. However, of the three - knee, hip, and hand OA - the evidence for OA of the hand is the most equivocal. Older studies have found an association between OA of the interphalangeal joints and overweight/obesity in males [51] and both males and females [52]. In one large cross-sectional study of the general population, obesity was found to be strongly associated with OA of the knees and the carpometacarpal joint and weakly associated with OA of the interphalangeal joints [44]. Some authors have suggested that the location of body fat may be more important in the development of hand OA than in other forms of the disease. For example, Hart and Spector found no effect of body fat distribution on OA diagnosis and suggested that the presence of central fat appeared to be more important to the relationship between OA and obesity [44]. However, some evidence suggests the opposite- that fat distribution may play a role in explaining the relationship between hand OA and weight. For example, Davis and colleagues found that radiological OA of the hand was associated with peripheral 
rather than central fat distribution in men, but not in women [53]. However, the paucity of studies that specifically examined the relationship between weight and hand OA make conclusions about the nature and strength of the association difficult.

\section{Summary: Relationship Between Overweight/Obesity and Osteoarthritis}

- Being overweight is a risk factor for development and progression of OA in the knee and hip, and possibly development of OA in the hand.

- Weight loss is recommended for overweight persons with OA.

- Even being slightly overweight may increase your risk for developing knee OA.

\section{Mechanisms: Explaining the Relationship between Pain and Overweight/Obesity}

With available evidence suggesting a likely relationship between increased BMI and a variety of pain conditions, the question remains as to why and how these relationships emerge. Mechanisms by which this link is hypothesized to develop generally include mechanical, structural, metabolic, and behavioral changes. Most likely, a combination of factors is responsible for the relationship-and these factors may contribute differently depending on the particular disorder.

In terms of mechanical-structural factors, several researchers have noted that obesity results in serious postural changes that affect loading on individual joints, and thus increased weight may result in long-term adverse effects on bones and joints [54]. Other hypothesized mechanical-structural hypotheses include increased loading [55], joint misalignment [56-57], structural changes [58], and decreased ambulation and conditioning [37-38] that result from overweight/obesity.

Metabolic factors have also been suggested as a possible link between overweight/obesity and pain disorders. Overweight/obese individuals have an increased risk of various metabolic disorders, thus they have increased vulnerability to the neuropathic disorders associated with conditions such as diabetes [59]. However, altered sensory thresholds have been shown in nondiabetic obese individuals, which suggests that obesity alone may increase one's risk for peripheral neuropathic disorders [60]. For example, obesity has been found to be a risk factor for carpal tunnel syndrome [61]. However, whether obesity is an independent risk factor for development of neuropathic pain disorders is not entirely clear. For example, De Block and colleagues found that BMI was not independently associated with neuropathy in a population of patients with type 1 diabetes [62]. Others have found that the incidence of neuropathy among those with type 1 diabetes who received care at specialty clinics was related to duration of disease and glycosylated hemoglobin values (the two major known determinants of neuropathy) and that, after controlling for these, higher BMI was one among many cardiovascular risk factors associated with cumulative incidence of neuropathy [63]. Some authors have proposed that altered pain sensitivity in obese individuals may be one pathway through which we can better understand the relationship between weight and pain. Differences in experimental measures of pain sensitivity have been found between obese and nonobese individuals, and in some studies, obese individuals were shown to be significantly more pain sensitive [64-65]. However, other studies in human and animal models have shown the opposite-decreased pain sensitivity in the obese [6667]. Finally, researchers have begun to examine common behavioral pathways that could contribute to the development of both disorders.

While much of the evidence reviewed for this article suggests overweight/obesity is a risk factor for development of certain pain conditions, equally possible is that pain could be a risk factor for weight gain. For example, individuals who experience ongoing pain may reduce their activity levels and, as a result, experience weight gain and eventual deconditioning, which may have the unwanted result of actually increasing pain. Specific lifestyle and psychosocial factors such as diet, activity level, health status, and smoking status may all provide shared pathways through which both obesity and pain develop.

However, any one of these factors alone is likely not a sufficient explanation for the relationships found between overweight/obesity and pain disorders. In some conditions, such as knee OA, mechanical-structural factors are more likely to play a larger role in understanding the cause-effect relationship between BMI and disease than other factors (such as metabolic and inflammatory effects of adiposity) because the effect of these on OA development in obese individuals is currently less well understood [68]. In other circumstances, an initial injury may lead to ongoing pain, reduced activity, and subsequent weight gain. It seems likely that patient subgroups exist in which the contribution of individual factors and the interplay between these factors varies according to multiple 
dimensions. However, given what little is currently understood about the role of metabolic versus mechanical-structural versus behavioral factors, much research remains to be done to better understand the role of each of these factors in clarifying the relationship between pain and overweight/obesity.

\section{Summary: Relationship Between Pain and Overweight/ Obesity}

Hypothesized mechanisms include-

- Mechanical.

- Structural.

- Metabolic.

- Behavioral changes.

- Most likely, a combination of factors.

- Factors may contribute differently depending on the particular disorder.

\section{Health-Related Quality of Life, Pain, and Overweight/ Obesity}

HRQoL has been defined as the effect of medical conditions on well-being, physical functioning, and mental functioning [69-70]. HRQoL represents the functional effects of an illness on the patient as perceived by the patient. As one of the aims of treating patients is to help them feel and function better, understanding the effect of both pain and overweight/obesity on HRQoL is an important first step in developing effective treatments that address their co-occurrence.

HRQoL has been measured via self-report in overweight/obese patients with various measures, including the Rand 36-item Health Survey, Health Status Questionnaire-12, EuroQoL, Goteborg Quality Life instrument, and most commonly, the Medical Outcomes Study 36item Short Form (SF-36) Heath Survey. In general, these measures assess mental, social, and physical functioning. With the exception of the Goteborg and EuroQoL, all the measures contain a bodily pain subscale, which will be the focal point of the studies discussed here.

\section{Bodily Pain Subscale Findings in Individuals with Higher Body Mass Index}

Generally, research to date suggests that overweight/ obese individuals report more pain on bodily pain subscales of HRQoL measures [71], but whether this relationship is direct or mediated by other factors is unclear. Overweight, obese, and morbidly obese individuals enrolled in either cohort or weight loss treatment studies were found to have significantly lower scores on the bodily pain subscale of the SF-36 [6] and Health Status Questionnaire-12 [72] compared with normal weight individuals. On the other hand, studies have found that overweight/obese women enrolled in a cohort study did not have significantly lower scores on the bodily pain subscale of the SF-36 compared with normal weight women [73] and depression, rather than BMI, explained low scores on the bodily pain subscale of the SF-36 in bariatric surgery candidates [74].

Some studies that have assessed HRQoL before and after weight loss treatment have shown a significant improvement in bodily pain scores following treatment [75-76], with one study demonstrating significant improvement through 4-year follow-up [75]. However, other studies that have examined lifestyle interventions, such as enrollment in Weight Watchers and increased physical activity [77], traditional aerobic activity versus lifestyle physical activity [78], and diet and exercise treatment versus control [79], found no significant effect on bodily pain subscale scores despite significant improvements in physical functioning. In particular, two methodological issues may help explain the varying results regarding the relationship between pain subscale scores and elevated BMI. First, body weight and height were not consistently measured across these studies. Some relied on self-reported weight and height, while others did not describe how this data was obtained. Self-reported weight and height are considerably less accurate than weight and height measured with a balance beam scale [80-81]. Second, these studies frequently did not assess pain at multiple time points but rather assessed pain at one time point only or, at best, immediately pre- and posttreatment. Pain intensity can vary throughout the day and also across different days [82]. Therefore, the lack of a significant relationship between pain subscale scores and high BMI in some studies may also be due in part to minimal or no pain on the day of assessment.

Overall, while overweight/obese individuals likely do have worse scores on pain subscales of HRQoL instruments, the direct effect of weight on these measurements is unclear. More research is needed to clarify the effect of other factors such as depression and health status along with weight on HRQoL outcomes. Additionally, while use of the bodily pain scale is useful in initial research, given what is already known about the likely relationship between increasing BMI and pain, its utility as a sole measure of pain-particularly within treatment outcome research-is limited and should be supplemented with more specific measures of pain. 


\section{Effect of Pain on Health-Related Quality of Life in Overweight/Obese Patients}

Some researchers have begun to study the effect of pain on HRQoL in obese individuals, and findings to date suggest the presence of pain in obese individuals may have an additive negative effect on HRQoL. One study investigated the effects of pain on HRQoL in a sample of 312 obese individuals who were seeking weight loss treatment [83]. Slightly less than half of these patients reported moderate or severe pain, and the most common types of pain reported included arthritis, chest pain, LBP, and headache. Obese patients who reported pain scored significantly lower on all domains/subscales (pain subscale not included) of the SF-36 compared with obese patients without pain. Similarly, in a sample of chronic pain patients from a university pain clinic, Marcus found that increased BMI was associated with comorbid pain disability, depression, and reduced physical functioning as measured by the SF-36 [84].

Other researchers have suggested that pain may mediate the relationship between increasing BMI and HRQoL such that the effects of high BMI on HRQoL are magnified in the presence of pain [85]. For example, Sendi and colleagues found that for patients with class II and class III obesity, while higher BMI alone was not directly associated with lower quality of life, presence of LBP in the context of obesity was significantly associated with reduced HRQoL as measured by the EuroQoL questionnaire [86]. Finally, evidence exists that HRQoL improved with weight loss treatment for obese patients who were experiencing pain. In a study of 218 morbidly obese patients who received lapband surgery, 72 percent reported arthritis/joint pain or discomfort prior to surgery [75]. One year after surgery, patients who had arthritis/joint pain improved significantly more than patients without pain on all but one of the eight SF-36 domains/subscales. These preliminary studies suggest that pain has a significant effect on overall HRQoL in obese patients, but whether the same is true for overweight patients is unknown. The effect of pain on HRQoL in patients with higher BMIs appears to be a fruitful area for continued study.

Summary: Health-Related Quality of Life, Pain, and Overweight/Obesity

- Overweight/obese individuals have lower scores on bodily pain scales compared with normal weight individuals.
- Pain has a significant negative effect on HRQoL in obese patients.

- Individuals who struggle with both pain and weight report lower HRQoL than those who experience only one of the two.

\section{Effect of Treatment on Pain and Weight Outcomes}

Weight loss appears to positively affect pain outcomes both in terms of lowering the risk of developing problems, such as musculoskeletal pain and $\mathrm{OA}$, and reducing pain-related distress and disability [87-88]. A variety of different weight-reduction treatment approaches has been evaluated. Selection of the particular approach depends, in part, on the characteristics of the sample under investigation. Surgical intervention, for example, is generally used only for individuals who are severely obese, whereas components of cognitive-behavioral therapy are often incorporated into weight reduction treatment with overweight and obese individuals.

\section{Surgical Intervention for Weight Reduction}

Bariatric surgery refers to operations performed to facilitate weight loss in severely obese individuals [89]. The procedures result in weight loss by decreasing food intake (restrictive procedures), disrupting the digestive process (malabsorptive procedures), or a combination of the two [90]. Bariatric surgery appears to have a positive effect on pain outcomes. Research suggests that surgical intervention may reduce the risk of developing musculoskeletal pain as well as facilitate recovery from it. Some research suggests that compared with nonsurgical obesity treatment involving dietary changes and behavior modification, surgical intervention results in a greater reduction of musculoskeletal pain [91]. For example, in a large sample of 2,010 surgically treated obese patients and 2,037 obese patients who received behavioral weight-reduction treatment, researchers compared the incidence of and recovery from musculoskeletal pain. Results revealed a reduced incidence of musculoskeletal pain as well as enhanced recovery from pain in both men and women who received surgical intervention for obesity compared with those who received nonsurgical behavioral weightmanagement intervention. Results were related to the degree of weight change and, impressively, were maintained over a 6-year follow-up period.

Other research supports the conclusion that surgical weight reduction positively affects pain outcomes in the majority of recipients postoperatively. However, in many of these studies the extent of weight loss was not strongly associated with pain outcome. In a study of morbidly obese 
patients with LBP, 19 of 29 patients with preoperative LBP reported complete resolution of symptoms 2 years after surgery [92]. The remaining 10 individuals reported notable improvement in symptoms and reduction in pain medication use. The extent of weight loss did not differ significantly between those reporting full versus partial improvement. In the same sample of 29 patients, researchers also noted significant postoperative improvement in several validated measures of functional status [93]. Similar findings on pain remission and reduction were demonstrated in an earlier study of morbidly obese individuals with musculoskeletal pain [94]. Finally, Dixon et al. assessed HRQoL, including bodily pain, in 218 morbidly obese patients both pre- and post-weight-reduction surgery [75]. Results revealed significant improvement in all aspects of HRQoL, with levels comparable with community norms at 4-year follow-up. The extent of weight loss was not a strong predictor of improved bodily pain or other aspects of HRQoL. Interestingly, the resulting effect on quality of life varied somewhat by the type of pain condition. Patients with lower-limb pain (e.g., foot, knee) reported significantly greater improvement in quality of life than those with LBP, often meeting or exceeding community norms.

\section{Lifestyle Intervention/Behavioral Weight-Loss Treatment}

Lifestyle interventions encourage weight loss through diet, exercise, and behavior and thought modification. Common components of behavioral interventions include education, goal setting, problem solving, and cognitive restructuring. Research on the effect of nonsurgical weight reduction on pain and its associated outcomes is promising. For example, although little is known about the relationship between fibromyalgia syndrome (FMS) and overweight/obesity, some research has examined the effect of treatment for overweight/obesity on outcomes for FMS. In a pilot study, Shapiro et al. examined the relationship between weight change and FMS symptoms and the effect of behavioral weight loss treatment on FMS [95]. The LEARN program for weight control was used, which emphasizes diet, physical activity, and stimulus control (e.g., shopping from a list, eating in only one place) [96]. Thirty-one women participated in the treatment. Participants lost an average of $>9 \mathrm{lb}$ and reported significant pre- to posttreatment improvement in pain, pain interference, and HRQoL. Importantly, weight loss predicted improvement in FMS symptoms, pain interference, and body dissatisfaction.
Research has also examined the combination of verylow-energy diet (VLED) with behavioral weight management on pain-related outcomes [76]. One hundred participants completed 4 months of behavior modification based on the LEARN program that included 10 weeks on a VLED. Posttreatment results revealed significant weight loss and marked improvement in HRQoL, including physical functioning and bodily pain. The vast majority of participants struggled with weight-loss maintenance (as is common in most nonsurgical weight-loss interventions). However, a more encouraging finding revealed that at 2-year follow-up, retention of 10 percent or more of original weight loss was associated with improvement from baseline in several areas of HRQoL, including bodily pain. Given the frequency of some weight regain following weight-loss intervention, such findings are highly encouraging. The same group of researchers also conducted a randomized clinical trial (RCT) to compare the same weight management program (VLED and behavior modification; $n=19$ men) with a no intervention control group ( $n=19$ men) and examine within- and between-group differences in HRQoL at posttreatment and 4-month follow-up [79]. Although the treatment group evidenced improvement in several aspects of HRQoL and maintained an average weight loss of 13.9 percent of baseline weight, improvement in bodily pain was only temporary and no longer evident at 4-month follow-up. Similarly, Christensen and colleagues demonstrated that 8 weeks on a VLED combined with weekly behavioral therapy resulted in significant improvements in both weight and pain outcomes [97]. Their findings that a modest weight loss of 10 percent resulted in a 28 percent reduction in patients' report of OA symptoms suggest that even a relatively brief dietary intervention with modest results can have a significant effect on pain symptoms. However, long-term outcomes are not clear from these results and, thus, we do not know whether patients can maintain these gains over time.

To date, very few studies have examined the influence of a pain treatment on weight outcome and then only as part of a larger weight-loss intervention. Huang and colleagues examined the effects of weight reduction and electrotherapy on pain relief and functional improvement in 126 obese patients with knee OA [88]. Participants were divided into one of three treatment groups: weight reduction, weight reduction and electrotherapy (i.e., transcutaneous electric stimulation for pain relief), or electrotherapy alone. Weight reduction differed from some of the more conventional nonsurgical programs and included dietary counseling and prescription, exercise prescription, 
and auricular acupuncture. The latter involved the implantation of needles in specified locations on the ears, and participants were asked to press the implanted needles when hungry or before meals. Participants who received weight-management treatment as well as electrotherapy reported the greatest pain relief and demonstrated the highest functional improvement. Weight reduction of 15 percent of initial body weight resulted in clinically and statistically significant pain relief, whereas a 12 percent weight reduction resulted in acceptable functional status. The researchers concluded that incorporation of electrotherapy for pain control, when appropriate, may be an important adjunct to weight-management treatment [88].

Of the behavioral interventions, research most strongly supports the combination of diet- and exercise-based intervention in improving pain outcomes. The Arthritis, Diet, and Activity Promotion Trial (ADAPT) is the largest single blind RCT to date [98-99]. ADAPT was designed to examine the effectiveness of exercise and dietary intervention on improving pain outcomes in overweight/obese older adults (60 years of age and older) who were suffering from knee OA. Three hundred sixteen adults were randomized into diet only, exercise only, diet plus exercise, and healthy lifestyle (control) groups. Primary outcome variables included physical functioning, pain, and mobility. Although the dietonly group experienced greater weight loss compared with the healthy lifestyle group, no pain-related improvement was evident. In the exercise-only group, the only significant improvement was in mobility. However, the diet and exercise group had significant improvement in physical functioning, self-reported pain, mobility, and weight.

Earlier research also supports the importance of both diet and exercise. In their RCT of a weight control program for obese patients with knee OA, Toda and colleagues compared participants assigned to a weight control program that included a low-calorie diet, an appetite suppressant, a nonsteroidal anti-inflammatory drug, and instruction on a walking program $(n=22)$ with a control group that received a nonsteroidal anti-inflammatory drug and instruction on a walking program $(n=15)$ [100]. Those who received the weight treatment had significant decreases in weight and body fat compared with those in the control group. Results also revealed that increased physical activity and decreased body fat were more important in enhancing pain outcome than weight loss per se.

Methodological differences among studies make conclusions about the influence of weight loss treatment on pain tentative at best. One issue relates to the samples used in the research. Samples in the reviewed studies represented potentially very different populations, thus making comparisons across studies difficult. Whereas some researchers recruited based on overweight/obesity status [76], others selected individuals based on type or severity of pain condition in addition to weight [99-100].

Second, pain was examined as a secondary outcome variable in several of the studies. Although this approach is useful in exploratory research, future research could benefit from exploration of more systematic a priori hypotheses related to pain outcome. Treatment efficacy and effectiveness may vary by pain type, etiology, and/or site.

Variability also existed in intervention design across studies. Programs varied in length, intervention component(s), and presence/length of follow-up period. Several incorporated multiple components into treatment, which at times made isolation of the active treatment component(s) difficult. Systematic evaluation of treatment components as well as taking into account pain type may help clarify the relationship among weight, pain conditions, and painrelated outcomes. Messier and colleagues, for example, found that without exercise, weight loss alone did not lead to significant improvement in pain or mobility among overweight/obese individuals with knee OA [99].

An additional methodological issue relates to variability in definitions and assessment of pain and other outcome variables. Some studies used self-reported pain, while others relied on clinical assessment and diagnosis of severity or functional disability [88,100]. Even among those that used a similar methodology (e.g., self-report), definitions included 12 months of work-restricting pain [91], intensity/frequency of pain [92], or pain evaluated in the context of HRQoL [79].

Finally, several studies were cross-sectional in design or lacked comparison groups. When comparison groups were used, group characteristics often differed across studies (i.e., nonobese versus obese). Recent research has incorporated use of RCTs and lengthier follow-up periods to evaluate maintenance of change [98-99]. Continued systematic, hypothesis-driven research will help elucidate treatment effects on weight and pain.

\section{Relationship Between Weight Loss and Improved Pain Outcomes}

Several explanations for the relationship between weight loss and improved pain outcomes have been proposed. Possible mediators include decreased pressure on 
joints, increased energy, and increased ease in performing physically demanding tasks [90]. Possible intermediaries may also vary by type and/or location of pain. In addition, the psychological implications of weight loss may also help explain the relationship. Weight loss is associated with improved self-esteem and decreased depression, both of which may affect individuals' perceptions of pain. Interestingly, some studies do show that weight loss significantly predicts improvements in pain outcomes, even after controlling for depression [95]. However, to date little is known about possible mechanisms that may be responsible for the relationship between weight reduction and improved pain outcomes.

\section{Summary: Effect of Treatment on Pain and Weight Outcomes}

Overall, the literature suggests an association between weight loss and improved pain outcomes, but the strength of the relationship varies by study and perhaps etiology/type of pain. Results suggest that even modest weight loss may be beneficial and that the extent of weight loss is not necessarily predictive of enhanced pain outcomes. Body composition variables, such as body fat composition, may also affect the relationship between weight and pain. One clear finding is that dietary modification coupled with exercise appears most effective in enhancing outcomes in nonsurgical interventions. Given the importance of exercise in both weight loss maintenance and pain management more generally, weight reduction interventions should include a physical activity component that enhances the likelihood of positive outcomes in long-term weight loss and pain management. Finally, some limited evidence exists that a combination of weight loss and pain treatment strategies may result in the most overall improvement; however, more research is needed to determine how to most effectively target the co-occurrence of pain and overweight/ obesity.

In conclusion-

- Surgical weight loss results in improvement in pain with substantial weight loss.

- Behavioral interventions suggest even modest weight loss may be beneficial.

- Weight loss may reduce the risk of pain-related problems and also reduce disruption in terms of pain experience, distress, and disability.

\section{DISCUSSION}

Both pain and overweight/obesity are critical issues within the VHA. Research reviewed in this article suggests that half of patients who seek obesity treatment report moderate to severe pain. Cross-sectional studies show support for a relationship between obesity and several pain conditions, including OA [43-44] and possibly LBP [19-23]. Similarly, longitudinal studies suggest overweight/obesity early in life may be a risk factor for developing LBP [26] and OA [47], and some evidence supports a dose-response relationship between BMI and pain. The co-occurrence of pain and obesity seems to have an additive negative effect on HRQoL, a finding demonstrated both in patients who were seeking treatment for obesity [83] and in patients who were seeking treatment for chronic pain [84]. Some have suggested that the presence of pain may mediate the association between BMI and HRQoL [85]. Finally, observations from a few studies have demonstrated that treatments for obesity reduce pain secondary to weight loss [87-88]. While research to date has explored possible relationships between weight and pain, future research needs to further clarify these relationships through specific examination of common pathways and development of treatments that may target their co-occurrence. The remainder of this article focuses on specific steps that researchers can take to use what is already known about the relationship to move forward our understanding of weight and pain.

First, while we know that independently pain and overweight/obesity are significant concerns within the VHA, information on the prevalence of the comorbidity of the two conditions is lacking. Given the magnitude of both problems within the VHA, research should examine the prevalence of both conditions as a first step in identifying and treating what is likely a common, costly, and disabling comorbidity among veterans. These studies need to assess both the relationship between pain and weight more generally and also the relationship between specific pain diagnoses and weight-related variables. Indeed, similar questions should also be posed in nonveteran samples because little is known about the relationship of increasing weight to outcomes in specific pain diagnoses in any population. For example, only one identified study explored the effect of weight loss treatment on symptoms of FMS [95], yet very few studies to date have even explored the relationship between weight and a diagnosis of FMS. In general, little is known about the prevalence of various pain disorders within overweight/obese populations 
and conversely the prevalence of high BMI within various pain populations. While comparatively more is known about overweight/obesity in certain pain populations, such as those with OA and LBP, much less is known about the relationship to pain disorders such as FMS, rheumatoid arthritis, and headache.

Second, studies that examined the relationship between pain and overweight/obesity rarely defined pain in a clear, consistent, or sometimes even meaningful, manner. Thus, a comparison of findings across studies is difficult. We found definitions of pain that ranged from chronic to acute, occasional to recurrent, any pain experienced during the last week to any pain experienced in the last month, and so on. Frequently, these pain ratings were poorly defined and rarely described with standard labels such as "acute" or "chronic." Studies are needed that use definitions of pain that have clinical meaning and, when possible, are linked to diagnostic criteria. While certainly self-report data serve a useful purpose in explorative research, clinical assessment of pain complaints using established criteria is the ideal. Additionally, other important pain-relevant variables were frequently absent or poorly measured within this literature. Thus, little is known about pain disability/interference, pain quality, pain intensity, pain duration, and emotional functioning in overweight/obese patients who also experience pain. Several well-validated measures are available to address each of these areas; however, they have been used infrequently. Where possible, future research should incorporate meaningful definitions of pain duration from established clinical and/or diagnostic criteria, use clinical assessment rather than self-report, and examine a variety of pain-relevant variables.

Several of the currently available studies in this area include pain as one variable among many believed to be affected by overweight/obesity. While these may be useful for generating hypotheses about the possible relationship between the two, few studies to date examined the relationship between weight and pain with specific testable hypotheses. From current research, particularly in the areas of LBP and OA, evidence supports a relationship between weight and pain. Investigators now need to take the next step and pursue research driven by specific hypotheses regarding the nature of this relationship, causal pathways, and treatments likely to address their co-occurrence. While a number of mediating factors likely affect the relationship between weight and pain, both basic and applied research conducted with specific hypotheses and multilevel analyses are needed to fully examine and understand these factors.
Finally, while initial reports suggest that improved weight outcomes also result in improved pain outcomes, availability of literature on the effect of weight treatment on pain outcomes is somewhat limited and has been compromised by methodological difficulty. Furthermore, to date studies examining the effect of pain-related treatment on weight outcomes are lacking. Research that has examined pain treatments included within weight-loss protocols suggests that the addition of pain treatment results in significant pain relief and functional improvement for obese individuals experiencing pain [88]. These results suggest a promising future for investigators looking to develop treatments that simultaneously target both pain and obesity. Empirically validated treatments for obesity and pain share a common focus on increasing healthy lifestyle, decreasing the potential effect of risk factors, and increasing problemsolving; motivation; and self-efficacy [101-102]. Thus, it seems a reasonable hypothesis that a treatment focused on one condition may have a positive effect on another. However, despite the overlap in potential risk factors, similarity of interventional focus, and the likely comorbidity of obesity and pain, few studies demonstrate clearly whether and how one intervention can simultaneously result in improved outcomes for both overweight/obesity and pain.

Much work remains for investigators to increase understanding of treatment effects on pain and weight outcomes. If weight loss does result in a reduction of pain and improvement in pain-related outcomes, we do not know how much reduction in weight is required to see such a result. Also unknown are which specific kinds of treatments will be most effective in creating positive outcomes for both weight and pain — weight loss treatments, pain treatments, or a combination of both. Evidence suggests that obese patients with pain who receive rehabilitative therapy in conjunction with weight management treatment experience significantly more pain relief and functional improvement [88]. Similarly, other evidence suggests weight loss treatment may result in positive pain outcomes [87]. Future studies should continue to explore the effectiveness of targeting overweight/obese individuals experiencing pain with treatments tailored to address both complaints simultaneously. Researchers must continue to explore the intervention components, environmental supports, and individual characteristics that may contribute to and enhance longterm weight-loss maintenance as well as reduction in pain symptoms. An oft-stated criticism of behavioral weight management treatment is its failure to sustain weight-related change over time. Despite the reality of weight regain, the subsequent implications of weight maintenance on pain 
outcomes are under-studied. Future research should investigate the long-term consequences of possible weight regain on pain-related variables. More recent research has incorporated use of RCTs [98-99] and lengthier follow-up periods to evaluate maintenance of change. Researchers should also continue to pursue RCTs as one means by which to examine the relationship among weight, pain, and treatment outcomes. Where appropriate, pain-focused investigations should consider collecting height and weight as part of treatment protocols, and similarly weight-focused investigations should consider collecting pain-relevant data. Finally, the effectiveness of overweight/obesity treatments may vary by pain type, etiology, and/or site. More systematic evaluation of weight-related treatment components by pain type and site will help clarify the relationship among treatment type, pain conditions, and pain-related outcomes.

\section{CONCLUSIONS}

In conclusion, while available evidence suggests a likely relationship between overweight/obesity and pain, we have much to learn about the nature of this co-occurrence. Researchers need to use state-of-the-art measurement and rigorous scientific methodology to focus on expanding knowledge of the relationship between pain and obesity, while examining the subsequent effects on health-related outcomes, developing explanatory models that address the co-occurrence of overweight/obesity and pain, and evaluating and testing treatments that most effectively target this comorbidity and meet the needs of the ever-growing population of individuals struggling with both weight and pain.

\section{ACKNOWLEDGMENTS}

This material was unfunded at the time of manuscript preparation.

The authors have declared that no competing interests exist.

\section{REFERENCES}

1. Finkelstein EA, Fiebelkorn IC, Wang G. State-level estimates of annual medical expenditures attributable to obesity. Obes Res. 2004;12(1):18-24. [PMID: 14742838]
2. World Health Organization. Obesity: Preventing and managing the global epidemic. Geneva (Switzerland): World Health Organization; 1997.

3. Kerns RD, Otis J, Rosenberg R, Reid MC. Veterans' reports of pain and associations with ratings of health, health-risk behaviors, affective distress, and use of the healthcare system. J Rehabil Res Dev. 2003;40(5):371-79. [PMID: 15080222]

4. Calle EE, Thun MJ, Petrelli JM, Rodriguez C, Heath CW Jr. Body-mass index and mortality in a prospective cohort of U.S. adults. N Engl J Med. 1999;341(15):1097-1105. [PMID: 10511607]

5. Ogden CL, Carroll MD, Curtin LR, McDowell MA, Tabak CJ, Flegal KM. Prevalence of overweight and obesity in the United States, 1999-2004. JAMA. 2006;295(13):1549-55. [PMID: 16595758]

6. Das SR, Kinsinger LS, Yancy WS Jr, Wang A, Ciesco E, Burdick M, Yevich SJ. Obesity prevalence among veterans at Veterans Affairs medical facilities. Am J Prev Med. 2005; 28(3):291-94. [PMID: 15766618$]$

7. Bigal ME, Liberman JN, Lipton RB. Obesity and migraine: A population study. Neurology. 2006;66(4):545-50. [PMID: 16354886]

8. Yunus MB, Arslan S, Aldag JC. Relationship between body mass index and fibromyalgia features. Scand J Rheumatol. 2002;31(1):27-31. [PMID: 11922197]

9. Escalante A, Haas RW, Del Rincon I. Paradoxical effect of body mass index on survival in rheumatoid arthritis: Role of comorbidity and systemic inflammation. Arch Intern Med. 2005;165(14):1624-29. [PMID: 16043681]

10. Gureje O, Von Korff M, Simon GE, Gater R. Persistent pain and well-being: A World Health Organization study in primary care. JAMA. 1998;280(2):147-51. [PMID: 9669787] Erratum in: JAMA. 1998;280(13):1142.

11. Stewart WF, Ricci JA, Chee E, Morganstein D, Lipton R. Lost productive time and cost due to common pain conditions in the U.S. workforce. JAMA. 2003;290(18):2443-54. [PMID: 14612481]

12. Manek NJ, MacGregor AJ. Epidemiology of back disorders: Prevalence, risk factors, and prognosis. Curr Opin Rheumatol. 2005;17(2):134-40. [PMID: 15711224]

13. Andersson GB. Epidemiological features of chronic lowback pain. Lancet. 1999;354(9178):581-85. [PMID: 10470716]

14. Stranjalis G, Tsamandouraki K, Sakas DE, Alamanos Y. Low back pain in a representative sample of Greek population: Analysis according to personal and socioeconomic characteristics. Spine. 2004;29(12):1355-60. [PMID: 15187638]

15. Kaila-Kangas L, Leino-Arjas P, Riihimaki H, Luukkonen $\mathrm{R}$, Kirjonen J. Smoking and overweight as predictors of hospitalization for back disorders. Spine. 2003;28(16): 1860-68. [PMID: 12923477] 
16. Wright D, Barrow S, Fisher AD, Horsley SD, Jayson MI. Influence of physical, psychological and behavioral factors on consultations for back pain. Br J Rheumatol. 1995; 34(2):156-61. [PMID: 7704462]

17. Han TS, Schouten JS, Lean ME, Seidell JC. The prevalence of low back pain and associations with body fatness, fat distribution and height. Int J Obes Relat Metab Disord. 1997;21(7):600-607. [PMID: 9226492]

18. Heliovaara M, Makela M, Knekt P, Impivaara O, Aromaa A. Determinants of sciatica and low-back pain. Spine. 1991; 16(6):608-14. [PMID: 1830689]

19. Andersen RE, Crespo CJ, Bartlett SJ, Bathon JM, Fontaine KR. Relationship between body weight gain and significant knee, hip, and back pain in older Americans. Obes Res. 2003;11(10):1159-62. [PMID: 14569039]

20. Michel A, Kohlmann T, Raspe H. The association between clinical findings on physical examination and self-reported severity in back pain. Results of a population-based study. Spine. 1997;22(3):296-304. [PMID: 9051892]

21. Deyo RA, Bass JE. Lifestyle and low-back pain. The influence of smoking and obesity. Spine. 1989;14(5):501-6. [PMID: 2524888]

22. Webb R, Brammah T, Lunt M, Urwin M, Allison T, Symmons D. Prevalence and predictors of intense, chronic, and disabling neck and back pain in the UK general population. Spine. 2003;28(11):1195-1202. [PMID: 12782992]

23. Fanuele JC, Abdu WA, Hanscom B, Weinstein JN. Association between obesity and functional status in patients with spine disease. Spine. 2002;27(3):306-12.

[PMID: 11805697]

24. Leboeuf-Yde C, Kyvik KO, Bruun NH. Low back pain and lifestyle. Part II-Obesity. Information from a population-based sample of 29,424 twin subjects. Spine. 1999; 24(8):779-84. [PMID: 10222529]

25. Aro S, Leino P. Overweight and musculoskeletal morbidity: A ten-year follow-up. Int J Obes. 1985;9(4):267-75. [PMID: 4066114]

26. Lake JK, Power C, Cole TJ. Back pain and obesity in the 1958 British birth cohort: Cause or effect? J Clin Epidemiol. 2000;53(3):245-50. [PMID: 10760633]

27. Garzillo MJ, Garzillo TA. Does obesity cause low back pain? J Manipulative Physiol Ther. 1994;17(9):601-4. [PMID: 7884330]

28. Leboeuf-Yde C. Body weight and low back pain. A systematic literature review of 56 journal articles reporting on 65 epidemiologic studies. Spine. 2000;25(2):226-37. [PMID: 10685488]

29. Mirtz TA, Greene L. Is obesity a risk factor for low back pain? An example of using the evidence to answer a clinical question. Chiropr Osteopat. 2005;13(1):2.

[PMID: 15967048]
30. Van den Bosch MA, Hollingworth W, Kinmonth AL, Dixon AK. Evidence against the use of lumbar spine radiography for low back pain. Clin Radiol. 2004;59(1):69-76. [PMID: 14697378]

31. Heliovaara M. Body height, obesity, and risk of herniated lumbar intervertebral disc. Spine. 1987;12(5):469-72. [PMID: 3629398]

32. Popkess-Vawter S, Patzel B. Compounded problem: Chronic low back pain and overweight in adult females. Orthop Nurs. 1992;11(6):31-35,43. [PMID: 1491878$]$

33. Bostman OM. Body mass index and height in patients requiring surgery for lumbar intervertebral disc herniation. Spine. 1993;18(7):851-54. [PMID: 8316883]

34. Toda Y, Segal N, Toda T, Morimoto T, Ogawa R. Lean body mass and body fat distribution in participants with chronic low back pain. Arch Intern Med. 2000;160(21): 3265-69. [PMID: 11088088]

35. Yip YB, Ho SC, Chan SG. Tall stature, overweight and the prevalence of low back pain in Chinese middle-aged women. Int J Obes Relat Metab Disord. 2001;25(6):887-92. [PMID: 11439304$]$

36. Croft PR, Rigby AS. Socioeconomic influences on back problems in the community in Britain. J Epidemiol Community Health. 1994;48(2):166-70. [PMID: 8189172]

37. Verbunt JA, Seelen HA, Vlaeyen JW, Van de Heijden GJ, Heuts PH, Pons K, Knottnerus JA. Disuse and deconditioning in chronic low back pain: Concepts and hypotheses on contributing mechanisms. Eur J Pain. 2003;7(1):9-21. [PMID: 12527313]

38. Yamakawa K, Tsai CK, Haig AJ, Miner JA, Harris MJ. Relationship between ambulation and obesity in older persons with and without low back pain. Int J Obes Relat Metab Disord. 2004;28(1):137-43. [PMID: 14557828]

39. Lawrence RC, Helmick CG, Arnett FC, Deyo RA, Felson DT, Giannini EH, Heyse SP, Hirsch R, Hochberg MC, Hunder GG, Liang MH, Pillemer SR, Steen VD, Wolfe F. Estimates of the prevalence of arthritis and selected musculoskeletal disorders in the United States. Arthritis Rheum. 1998;41(5):778-99. [PMID: 9588729]

40. Guccione AA, Felson DT, Anderson JJ, Anthony JM, Zhang Y, Wilson PW, Kelly-Hayes M, Wolf PA, Kreger BE, Kannel WB. The effects of specific medical conditions on the functional limitations of elders in the Framingham Study. Am J Public Health. 1994;84(3):351-58. [PMID: 8129049]

41. Peat G, McCarney R, Croft P. Knee pain and osteoarthritis in older adults: A review of community burden and current use of primary health care. Ann Rheum Dis. 2001; 60(2):91-97. [PMID: 11156538]

42. American College of Rheumatology Subcommittee on Osteoarthritis. Recommendations for the medical management of osteoarthritis of the hip and knee: 2000 update. American College of Rheumatology Subcommittee on 
Osteoarthritis Guidelines. Arthritis Rheum. 2000;43(9): 1905-15. [PMID: 11014340$]$

43. Hartz AJ, Fischer ME, Bril G, Kelber S, Rupley D Jr, Oken B, Rimm AA. The association of obesity with joint pain and osteoarthritis in the HANES data. J Chronic Dis. 1986;39(4):311-19. [PMID: 3958117]

44. Hart DJ, Spector TD. The relationship of obesity, fat distribution and osteoarthritis in women in the general population: The Chingford Study. J Rheumatol. 1993;20(2): 331-35. [PMID: 8474072]

45. Manninen P, Riihimaki H, Heliovaara M, Makela P. Overweight, gender and knee osteoarthritis. Int J Obes Relat Metab Disord. 1996;20(6):595-97. [PMID: 8782738]

46. Holmberg S, Thelin A, Thelin N. Knee osteoarthritis and body mass index: A population-based case-control study. Scand J Rheumatol. 2005;34(1):59-64. [PMID: 15903028]

47. Anderson JJ, Felson DT. Factors associated with osteoarthritis of the knee in the first national Health and Nutrition Examination Survey (HANES I). Evidence for an association with overweight, race, and physical demands of work. Am J Epidemiol. 1988;128(1):179-89. [PMID: 3381825]

48. Felson DT, Anderson JJ, Naimark A, Walker AM, Meenan RF. Obesity and knee osteoarthritis. The Framingham Study. Ann Intern Med. 1988;109(1):18-24. [PMID: 3377350]

49. Gelber AC, Hochberg MC, Mead LA, Wang NY, Wigley FM, Klag MJ. Body mass index in young men and the risk of subsequent knee and hip osteoarthritis. Am J Med. 1999;107(6):542-48. [PMID: 10625021]

50. Flugsrud GB, Nordsletten L, Espehaug B, Havelin LI, Engeland A, Meyer HE. The impact of body mass index on later total hip arthroplasty for primary osteoarthritis: A cohort study in 1.2 million persons. Arthritis Rheum. 2006;54(3):802-7. [PMID: 16508955]

51. Kellgren JH, Lawrence JS. Osteo-arthrosis and disk degeneration in an urban population. Ann Rheum Dis. 1958;17(4):388-97. [PMID: 13606727]

52. Acheson RM, Collart AB. New Haven survey of joint diseases. XVII. Relationship between some systemic characteristics and osteoarthrosis in a general population. Ann Rheum Dis. 1975;34(5):379-87. [PMID: 1221922]

53. Davis MA, Neuhaus JM, Ettinger WH, Mueller WH. Body fat distribution and osteoarthritis. Am J Epidemiol. 1990;132(4):701-7. [PMID: 2403110]

54. Fabris de Souza SA, Faintuch J, Valezi AC, Sant'Anna AF, Gama-Rodrigues JJ, De Batista Fonseca IC, De Melo RD. Postural changes in morbidly obese patients. Obes Surg. 2005;15(7):1013-16. [PMID: 16105399]

55. Rodacki AL, Fowler NE, Provensi CL, Rodacki Cde L, Dezan VH. Body mass as a factor in stature change. Clin Biomech (Bristol, Avon). 2005;20(8):799-805. [PMID: 16005554$]$
56. Felson DT, Goggins J, Niu J, Zhang Y, Hunter DJ. The effect of body weight on progression of knee osteoarthritis is dependent on alignment. Arthritis Rheum. 2004; 50(12):3904-9. [PMID: 15593215]

57. Sharma L, Lou C, Cahue S, Dunlop DD. The mechanism of the effect of obesity in knee osteoarthritis: The mediating role of malalignment. Arthritis Rheum. 2000;43(3): 568-75. [PMID: 10728750]

58. Ding C, Cicuttini F, Blizzard L, Jones G. Genetic mechanisms of knee osteoarthritis: A population-based longitudinal study. Arthritis Res Ther. 2005;8(1):R8. [PMID: 16356187]

59. Lean ME. Pathophysiology of obesity. Proc Nutr Soc. 2000; 59(3):331-36. [PMID: 10997648]

60. Miscio G, Guastamacchia G, Brunani A, Priano L, Baudo S, Mauro A. Obesity and peripheral neuropathy risk: A dangerous liaison. J Peripher Nerv Syst. 2005;10(4):354-58. [PMID: 16279984]

61. Stallings SP, Kasdan ML, Soergel TM, Corwin HM. A case-control study of obesity as a risk factor for carpal tunnel syndrome in a population of 600 patients presenting for independent medical examination. J Hand Surg [Am]. 1997;22(2):211-15. [PMID: 9195416]

62. De Block CE, De Leeuw IH, Van Gall LF. Impact of overweight on chronic microvascular complications in type 1 diabetic patients. Diabetes Care. 2005;28(7):1649-55. [PMID: 15983315]

63. Tesfaye S, Chaturvedi N, Eaton SE, Ward JD, Manes C, Ionescu-Tirgoviste C, Witte DR, Fuller JH, EURODIAB Prospective Complications Study Group. Vascular risk factors and diabetic neuropathy. N Engl J Med. 2005; 352(4):341-50. [PMID: 15673800]

64. McKendall MJ, Haier RJ. Pain sensitivity and obesity. Psychiatry Res. 1983;8(2):119-25. [PMID: 6574530]

65. Pradalier A, Willer JC, Boureau F, Dry J. Relationship between pain and obesity: An electrophysiological study. Physiol Behav. 1981;27(6):961-64. [PMID: 7335814]

66. Khimich S. Level of sensitivity of pain in patients with obesity. Acta Chir Hung. 1997;36(1-4):166-67. [PMID: 9408331]

67. Ramzan I, Wong BK, Corcoran GB. Pain sensitivity in dietary-induced obese rats. Physiol Behav. 1993;54(3): 433-35. [PMID: 8415933

68. Powell A, Teichtahl AJ, Wluka AE, Cicuttini FM. Obesity: A preventable risk factor for large joint osteoarthritis which may act through biomechanical factors. Br J Sports Med. 2005;39(1):4-5. [PMID: 15618330]

69. Colditz GA. Economic costs of obesity. Am J Clin Nutr. 1992;55(2 Suppl):S503-7. [PMID: 1733119]

70. Fontaine KR, Cheskin LJ, Barofsky I. Health-related quality of life in obese persons seeking treatment. J Fam Pract. 1996;43(3):265-70. [PMID: 8797754$]$ 
71. Yancy WS, Olsen MK, Westman EC, Bosworth HB, Edelman D. Relationship between obesity and health-related quality of life in men. Obes Res. 2002;10(10):1057-64. [PMID: 12376587]

72. Yan LL, Daviglus ML, Liu K, Pirzada A, Garside DB, Schiffer L, Dyer AR, Greenland P. BMI and healthrelated quality of life in adults 65 years and older. Obes Res. 2004;12(1):69-76. [PMID: 14742844]

73. Brown WJ, Mishra G, Kenardy J, Dobson A. Relationships between body mass index and well-being in young Australian women. Int J Obes Relat Metab Disord. 2000; 24(10):1360-68. [PMID: 11093300]

74. Fabricatore AN, Wadden TA, Sarwer DB, Faith MS. Health-related quality of life and symptoms of depression in extremely obese persons seeking bariatric surgery. Obes Surg. 2005;15(3):304-9. [PMID: 15826461]

75. Dixon JB, Dixon ME, O’Brien PE. Quality of life after lapband placement: Influence of time, weight loss, and comorbidities. Obes Res. 2001;9(11):713-21. [PMID: 11707538]

76. Kaukua J, Pekkarinen T, Sane T, Mustajoki P. Healthrelated quality of life in obese outpatients losing weight with very-low-energy diet and behaviour modificationA 2-y follow-up study. Int J Obes Relat Metab Disord. 2003;27(10):1233-41. [PMID: 14513072]

77. Rippe JM, Price JM, Hess SA, Kline G, DeMers KA, Damitz S, Kreidieh I, Freedson P. Improved psychological well-being, quality of life, and health practices in moderately overweight women participating in a 12-week structured weight loss program. Obes Res. 1998;6(3):208-18. [PMID: 9618125]

78. Fontaine KR, Barofsky I, Andersen RE, Bartlett SJ, Wiersema L, Cheskin LJ, Franckowiak SC. Impact of weight loss on health-related quality of life. Qual Life Res. 1999;8(3):275-77. [PMID: 10472159]

79. Kaukua J, Pekkarinen T, Sane T, Mustajoki P. Healthrelated quality of life in WHO class II-III obese men losing weight with very-low-energy diet and behaviour modification: A randomized clinical trial. Int J Obes Relat Metab Disord. 2002;26(4):487-95. [PMID: 12075575]

80. Jacobson BH, De Bock DH. Comparison of body mass index by self-reported versus measured height and weight. Percept Mot Skills. 2001;92(1):128-32. [PMID: 11322576]

81. Santillan AA, Camargo CA. Body mass index and asthma among Mexican adults: The effect of using self-reported vs measured weight and height. Int J Obes Relat Metab Disord. 2003;27(11):1430-33. [PMID: 14574357]

82. Sorbi MJ, Peters ML, Kruise DA, Maas CJ, Kerssens JJ, Verhaak PF, Bensing JM. Electronic momentary assessment in chronic pain I: Psychological pain responses as predictors of pain intensity. Clin J Pain. 2006;22(1):55-66. [PMID: 16340594]

83. Barofsky I, Fontaine KR, Cheskin LJ. Pain in the obese: Impact on health-related quality of life. Ann Behav Med. 1997;19(4):408-10. [PMID: 9706368]
84. Marcus DA. Obesity and the impact of chronic pain. Clin J Pain. 2004;20(3):186-91. [PMID: 15100595]

85. Heo M, Allison DB, Faith MS, Zhu S, Fontaine KR. Obesity and quality of life: Mediating effects of pain and comorbidities. Obes Res. 2003;11(2):209-16. [PMID: 12582216]

86. Sendi P, Brunotte R, Potoczna N, Branson R, Horber FF. Health-related quality of life in patients with class II and class III obesity. Obes Surg. 2005;15(7):1070-76. [PMID: 16105410]

87. Felson DT, Zhang Y, Anthony JM, Naimark A, Anderson JJ. Weight loss reduces the risk for symptomatic knee osteoarthritis in women. The Framingham Study. Ann Intern Med. 1992;116(7):535-39. [PMID: 1543306]

88. Huang $\mathrm{MH}$, Chen $\mathrm{CH}$, Chen TW, Weng MC, Wang WT, Wang YL. The effects of weight reduction on the rehabilitation of patients with knee osteoarthritis and obesity. Arthritis Care Res. 2000;13(6):398-405. [PMID: 14635316]

89. Torpy JM, Burke A, Glass RM. JAMA patient page. Bariatric surgery. JAMA. 2005;294(15):1986. [PMID: 16234505]

90. Weight-control Information Network [homepage on the Internet]. Bethesda (MD): National Institute of Diabetes and Digestive and Kidney Diseases; [updated 2007 Jan 17; cited 2006 May 5]. Gastrointestinal surgery for severe obesity; [about 1 screen]. Available from: http://win.niddk.nih.gov/ publications/gastric.htm.

91. Peltonen M, Lindroos AK, Torgerson JS. Musculoskeletal pain in the obese: A comparison with a general population and long-term changes after conventional and surgical obesity treatment. Pain. 2003;104(3):549-57.

[PMID: 12927627]

92. Melissas J, Volakakis E, Hadjipavlou A. Low-back pain in morbidly obese patients and the effect of weight loss following surgery. Obes Surg. 2003;13(3):389-93. [PMID: 12841899]

93. Melissas J, Kontakis G, Volakakis E, Tsepetis T, Alegakis A, Hadjipavlou A. The effect of surgical weight reduction on functional status in morbidly obese patients with low back pain. Obes Surg. 2005;15(3):378-81. [PMID: 15826473]

94. McGoey BV, Deitel M, Saplys RJ, Kliman ME. Effect of weight loss on musculoskeletal pain in the morbidly obese. J Bone Joint Surg Br. 1990;72(2):322-23. [PMID: 2138158]

95. Shapiro JR, Anderson DA, Danoff-Burg S. A pilot study of the effects of behavioral weight loss treatment on fibromyalgia symptoms. J Psychosom Res. 2005;59(5):275-82. [PMID: 16253617]

96. Brownell KD. The LEARN program for weight control. 7th ed. Dallas (TX): American Health Publishing Company; 1998.

97. Christensen R, Astrup A, Bliddal H. Weight loss: The treatment of choice for knee osteoarthritis? A randomized trial. Osteoarthritis Cartilage. 2005;13(1):20-27.

[PMID: 15639633] 
98. Miller GD, Rejeski WJ, Williamson JD, Morgan T, Sevick MA, Loeser RF, Ettinger WH, Messier SP, ADAPT Investigators. The Arthritis, Diet, and Activity Promotion Trial (ADAPT): Design, rationale, and baseline results. Control Clin Trials. 2003;24(4):462-80.

[PMID: 12865040]

99. Messier SP, Loeser RF, Miller GD, Morgan TM, Rejeski WJ, Sevick MA, Ettinger WH Jr, Pahor M, Williamson JD. Exercise and dietary weight loss in overweight and obese older adults with knee osteoarthritis: The Arthritis, Diet, and Activity Promotion Trial. Arthritis Rheum. 2004; 50(5):1501-10. [PMID: 15146420]

100. Toda Y, Toda T, Takemura S, Wada T, Morimoto T, Ogawa R. Change in body fat, but not body weight or metabolic correlates of obesity, is related to symptomatic relief of obese patients with knee osteoarthritis after a weight con- trol program. J Rheumatol. 1998;25(11):2181-86.

[PMID: 9818662]

101. Morley S, Eccleston C, Williams A. Systematic review and meta-analysis of randomized controlled trials of cognitive behavior therapy and behavior therapy for chronic pain in adults, excluding headache. Pain. 1999;80(1-2):1-13.

[PMID: 10204712]

102. North American Association for the Study of Obesity. The practical guide: Identification, evaluation, and treatment of overweight and obesity in adults. Bethesda (MD): National Institutes of Health; 2000.

Submitted for publication June 1, 2006. Accepted in revised form September 18, 2006. 